\title{
Mars Commercial Rover Payload Services
}

White Paper Submission for the 2023-2032 Planetary Science and Astrobiology Decadal Survey

\author{
Author \\ Paul B Niles \\ NASA Johnson Space Center \\ paul.b.niles@nasa.gov; (281) 816-7780 \\ Abigail Fraeman \\ Jet Propulsion Laboratory/California Institute of Technology \\ Steve Ruff \\ Arizona State University
}

Co-Signers:

Nathan Barba, Jet Propulsion

Laboratory/Caltech

Susan Harkins Bertsch, NASA Johnson

Space Center

Janice Bishop, SETI Institute, Mountain

View, CA

Mackenzie Day, University of California Los

Angeles

Michael Evans, NASA Johnson Space

Center

Kenton Fisher, NASA Johnson Space

Center

David Flannery, Queensland University of

Technology, Australia

Timothy Glotch, Stony Brook University,

NY USA
Timothy A. Goudge, The University of Texas at Austin

Laura Kerber, Jet Propulsion Laboratory/Caltech University

Jack Mustard, Brown University,

Providence, RI USA

Charity Phillips-Lander, Southwest

Research Institute

Deanne Rogers, Stony Brook University, NY USA

Adam Schilffarth, Xplore Inc.

Sam Spencer, Etiam Engineering Australia 


\section{Executive Summary}

As the new decade begins, new programmatic strategies to conduct more frequent, lower cost missions are beginning to be applied to deep space robotic science missions, such as the Commercial Lunar Payload Services (CLPS) program. These new strategies include moving from cost-plus contracts towards fixed price contracts, commercial contractors increasingly sharing in development costs, finding launch opportunities through ride sharing and comanifesting payloads, and making use of smallsats and other spacecraft with off-the-shelf hardware. While these new practices are now being applied in many areas across NASA, they are not yet being widely implemented in the Mars exploration program, where large bespoke missions have become the dominant programmatic strategy. As the decadal survey for planetary science in the 2020's begins its deliberations, it should consider how programmatic strategies that emphasize lower cost, more frequent missions to Mars can provide groundbreaking science return and enable the beginning of a new age in Martian exploration[1].

Lower cost, less complex, and more frequent missions to Mars can have substantial scientific impact that perhaps rivals the much larger and more complex missions that have begun to define the Mars exploration program. Resolving the most important questions in Mars science demands the exploration of its geologic diversity to separate local trends from truly global processes and form a chronology of events. Previously, lower cost solar powered rovers have proven to be a successful strategy for Mars exploration, addressing habitability and astrobiological questions from multiple landing sites and diverse environments on Mars. This paper proposes a commercial payload Mars exploration strategy that could increase the number of available opportunities to either land on or orbit Mars and generate substantial new science using a fleet of commercially provided rovers.

\section{Background}

The Mars Exploration Program has made tremendous strides since the mid-1990s. Seminal discoveries include discovery of a sedimentary rock record, discovery of organics in rocks and soils, and recognition that Mars hosted over a dozen types of aqueous, potentially habitable environments. These include crater lakes of various ages and pH's, exhumed volcanic and impact hydrothermal systems of diverse chemistries and ages, and soil horizons, which on Earth, represent habitable and inhabited environments.

Furthermore, these environments all record information on timing of aqueous activity, global geochemical fluxes, and Martian processes. We do not yet understand the controls on these environments in time and space. The sheer diversity and number of potential landing sites that have been identified cannot possibly be addressed by only one or two large complex missions per decade. Without a broad-based exploration of the planet, individual discoveries lack context. Targeted missions to explore particular sets of Martian planetary science objectives are needed 
to address major outstanding scientific questions that are critical to understanding the evolution of the planet and its potential for hosting life.

There are at least 8 reasons why a low cost, multiple mission approach would be beneficial for the Mars Exploration Program[3]: Multiple missions exploring multiple exciting landing sites will substantially increase the Scientific Return of the Mars Program. Many of the major questions about Mars that have been raised during the past $\sim 5$ decades of Mars exploration remain unanswered. Orbital discoveries of sulfates, phyllosilicates, carbonates, and chloride salts require ground truthing to establish an historical and geological context. Multiple lower cost missions enhance the potential for Breakthrough Discoveries. The more areas of the planet that are explored, the more likely it is to find surprising things, and as the discovery of plate tectonics on Earth shows, multiple lines of evidence from across the planet will be required to set a new paradigm. Multiple lower cost missions provide the opportunity to increase

Programmatic Risk Tolerance. A critical weakness in the current Mars Exploration Program is that a single mission failure would endanger the entire program. This drives cost higher than it should be and discourages innovation. A program of smaller missions would provide substantial Budget Flexibility, spreading the costs over time and helping to supplement the Mars exploration program with lower cost missions. The economies of scale and flexibility realized from the small, multiple mission approach could be helpful for accomplishing the goals of NASA's Mars exploration efforts. The multiple mission approach would substantially drive down Cost Risk by utilizing off the shelf equipment, fixed price contracts, higher risk tolerance, and contractor cost-sharing. A higher cadence of Mars missions would increase the number of payload opportunities and drive International Cooperation. Contributed payloads from international partners would help fill out manifests and could enhance science return. Finally, multiple lower cost missions to Mars would provide for enhanced Technology Development. The costs and risks incurred by testing new technologies would be mitigated by the increased number of mission opportunities.

\section{Commercial Rover Exploration}

The advancements of technology and capabilities in outer space by NASA and the commercial industry have presented a wealth of new opportunities for Mars exploration that would substantially increase the science return of the program. Here we outline a potential programmatic strategy that would take advantage of recent developments and could lower the cost and increase the cadence of missions to Mars while providing a robust science return.

\section{Mars Commercial Payload Services Program}

Based on the preliminary indications of success in the Commercial Lunar Payload Services (CLPS) program, a Mars commercial payload services program has extraordinary potential. As an anchor tenant for the first several lunar missions, NASA has selected 3 small landers to carry payloads to the Moon for less than $\$ 100$ million each along with a much larger lander to carry a 
NASA built rover to explore for volatiles. The indefinite delivery/indefinite quantity contracting mechanism driven by firm fixed price task orders has incentivized substantial private investment and cost sharing and NASA stands to benefit from having up to 4 distinct flight proven systems for delivering payloads to the Moon before 2024. Not all of these missions may succeed but the success of any one of them will be a boon to lunar exploration as subsequent missions have the potential to continue driving the cost downwards. Lunar missions can be a powerful proving ground for Mars missions where many important technologies and capabilities can be tested and matured.

There are several concerns for applying the CLPS model to Mars. Since Mars is farther away and requires more complicated EDL, the missions are likely to be more expensive. Furthermore there are fewer perceived commercial opportunities at Mars because of its distance from Earth so it is almost certain that NASA will have to be an anchor tenant for a Mars commercial payload program. Until the first lunar CLPS missions fly, the degree of commercial interest in purchasing payload space on lunar landers will be difficult to determine. However, there is a possibility that NASA remains as an anchor tenant for the foreseeable future in the CLPS program. This is not necessarily a bad outcome especially if mission costs remain low for future flights. Similarly for Mars, NASA as an anchor tenant is not a deal breaker. While the increased complexity of Mars missions may substantially inflate initial Mars commercial missions - a steady commitment to the program and a high flight rate should bring those costs back down. Mars also provides a high potential for commercial opportunities such as ISRU. In fact, the distance from the Earth actually makes fuel acquired through ISRU more desirable since it wouldn't have to compete with water delivered from Earth. As launch costs continue to go down, lunar ISRU will need to compete with water delivered from Earth and may never become competitive.

\section{Mars Commercial Rover Payload Services (MCRPS)}

Solar powered rover exploration of Mars has been shown by the Mars Exploration Rovers (MER) to be an extraordinarily successful means for performing planetary science. Solar powered rovers have the potential for long mission durations and long traverse distances (> 28 miles). Solar powered rovers are small enough to accommodate different EDL technologies, and robust enough to carry useful science instrumentation.

In contrast to the CLPS program, we propose here that the Mars commercial program request vendors to supply both the lander and the rover. Thus NASA would be only supplying science instruments to be incorporated on vendor supplied rovers. This serves to keep costs low and decreases complexity for fixed price contracts.

A critical component for lowering costs of Mars rover exploration is by achieving a semblance of economies of scale in rover production. The two MER rovers together cost 800 million dollars to send to Mars in 2003. However, because they were built without consideration for future production, parts are no longer available and building just one MER rover today would likely 
cost much more than their previous price tag. This is because NASA builds bespoke science equipment and does not consider driving down costs for future missions as there is no apparent incentive. Commercial companies in a fixed price environment have quite different motivations and commercially provided rovers will benefit from utilization of commercially off the shelf parts and economies of scale as multiple missions are planned for. Therefore having the rover as part of the vendor supplied equipment is key for keeping costs low.

Intuitively it might appear that a vendor supplied lander and NASA supplied rover would provide the least complexity for fixed price contracts, but this underestimates NASA's ability to design complex and bespoke rovers. The most complex set of requirements provided by NASA for a CLPS mission has been the task order for the NASA built VIPER rover which was awarded to Astrobotic[2]. The sheer variety of different rover types that can be designed and built by NASA would each require their own set of complex requirements for delivery to the martian surface and deployment after landing. However, if the vendors are delivering a rover and lander of their own design, then these interface requirements are greatly simplified, and the only ones remaining are for the instruments.

Commercial companies will have the flexibility to operate the rovers in ways that help drive operation costs down, especially during extended missions. There are many strategies that commercial companies can utilize that NASA cannot pursue. Some of these strategies could include increased student participation and expansion of scientific investigation of Mars into communities that traditionally have little access.

\section{Instrument Selection for the Mars Commercial Rover Payload Services Program}

Clearly not all science instruments would be appropriate for a fixed price payload delivery program. Some instruments require complex interfaces with a rover that substantially alter its fundamental design. A good example are the sampling payloads included on MSL and Mars2020 which drove the design of a substantial robotic arm and thus the overall mass of the mission. Payloads requiring complex sampling or deployment capabilities may not be appropriate for this type of payload delivery program. Instead, science instruments with simple interface requirements like cameras and other remote sensing instruments would be ideal. NASA may be able to design a robotic arm with modular payload slots that could also be offered as a means for executing surface science.

A successful Mars commercial payload services program would include an instrument development funding line that prioritised instruments suitable for this type of delivery mechanism. By focusing on solar powered rovers with less complex interface requirements, more substantial limits would be placed on instrument designers. This may in fact be beneficial to the mission as it could avoid science capability creep where evermore complex and expensive instruments are designed to make more challenging measurements, rather than focusing on the most important ones identified by the science community. These instruments 
could also be included in other missions of opportunity including international and human exploration missions.

\section{Leveraging Commercial Infrastructure Support}

The Mars exploration program may save money by contracting out communications to commercial companies. Enabling opportunities for commercial companies to sell bandwidth to NASA may substantially enhance the number of surface and orbital opportunities NASA can pursue. As interest in Mars exploration increases across the world both internationally and commercially, communications provide a compelling commercial avenue and NASA could benefit greatly by enabling opportunities in this market. In addition to Mars rovers, the most successful science platforms at Mars have been orbiters. There remain substantial new ideas for orbital instruments that could provide large science returns and could accompany a commercial orbiter focused around communications to Earth. These types of missions could also benefit from the increasing opportunities to ride-along with other launches.

The success of the MARCO cubesats in association with the Insight mission has demonstrated that substantial benefit can be gained from ridealong spacecraft. The available mass margin in nearly every launch should not be passed up and can be leveraged in many different ways. The use of ESPA rings and similar equipment to deploy smaller spacecraft after the primary payload has been delivered is highly encouraged and NASA should consider enabling more Mars opportunities using programs like SIMPLEX. Furthermore, in the same way that launch vehicles have created accomodations for additional payloads - Mars entry descent and landing systems might be designed to accommodate additional ridealong payloads for delivery to the surface. This could enable a wide variety of stand-alone scientific investigations such as balloons, drones, small rovers, and surface deployed seismic instruments.

\section{Discovery and New Frontiers}

As they are currently constituted, the highly successful Discovery and New Frontiers programs provide essential opportunities to explore lower profile destinations outside of the Moon and Mars but do not leverage the new commercial spacecraft providers as well as they could. Increasing pressure to include Moon and Mars destinations in the Discovery and New Frontiers programs will inevitably either dilute those programs with more conventional missions or provide a roadblock to important Moon and Mars science as they cannot compete in novelty. Furthermore Discovery and New Frontiers missions are pitched as complete end to end bespoke missions and as such they miss opportunities to entertain commercial platforms for payload delivery. Since Mars and the Moon are common destinations for planetary missions, it makes sense to take advantage of this to help lower costs and increase mission frequency.

Therefore it is proposed here that Mars and the Moon remain outside of the Discovery and New Frontiers programs to focus on destinations with novel science goals where those missions have tended to shine. This allows for the continued funding of commercial payload services 
programs to each body to conduct efficient and frequent exploration of the multitude of landing sites that have been identified to be of scientific interest.

\section{Scientific Return on Mars Rover Commercial Payload Services}

An obvious potential drawback to sending lower cost missions to Mars would be a loss of capability. However the types of low cost missions with simple science payloads that could be flown and have already flown shows that they can provide incredible returns especially when they are paired with a higher flight rate. Several major science questions have not yet been addressed by landed missions including: 1) the formation of the martian dichotomy and early evolution of the planet 2) the age, history, and evolution of the Tharsis bulge, 3) the age, history, and evolution of Mars polar deposits, 4) exploration of low latitude glacial deposits, 5) the age, composition and formation of chloride deposits, 6 ) exploration of valley networks, 7 ) exploration of chaos terrain and outflow channels, 8) monitoring of recurrent slope linear, and many more.

The Mars Exploration Rovers showed that solar powered rovers with relatively small payloads can do incredible science. Solar powered rovers with traverse distances of at least $10 \mathrm{~km}$ and payload capacities of less than $10 \mathrm{~kg}$ are extraordinarily powerful agents of scientific discovery and are capable of addressing a large number of high priority scientific investigations.

Instruments with common interfaces could include cameras (IR, VIS, multispectral), geophysical equipment (thumper, geophones, penetrator), meteorological instruments (wind, direction, density, humidity, temperature), and contact instruments (APXS, Mossbauer, XRF). Increasing landing accuracy capabilities $(<100 \mathrm{~m})$ that are now becoming a typical requirement for CLPS missions opens up large portions of the planet for exploration.

\section{Implementation Recommendations}

The past practice of only developing highly customized payloads alongside space vehicles for science missions creates an environment where near flight ready instruments and payloads are rare, and typically development needed to take instruments from lower TRL to flight only occurs when a mission gets funded. This creates challenges for achieving high science return on missions of opportunity like a commercial rover mission described above. Similar opportunities may be available for other missions like the SpaceX starship and human exploration missions where substantial mass may be available. In the current environment it will be difficult to capitalize on these types of missions because the payload and the science mission are so closely coupled.

Having instruments and payloads "on the shelf" for upcoming opportunities can substantially enhance science return on missions of opportunity. Commercial mars rovers outfitted with NASA instruments can be delivered to the surface by themselves, or could be included in a 
larger mission like what may be available on upcoming SpaceX, or Human Exploration missions.

The CLPS program provides a great opportunity to better understand how a Mars Commercial Payload Services program might work and what it needs to be successful. Maximizing science return on a commercial mission is going to be challenging, and understanding the types of missions that work and don't work in this model should help better design an overall Mars exploration architecture. Certainly any implementation of the type of program described in this white paper should incorporate lessons learned as the CLPS program moves forward as well as being responsive to developments in the human exploration program.

\section{References}

[1] B. M. Jakosky et al., "Mars, The Nearest Habitable World - A Comprehensive Program for Future Mars Exploration - Preliminary Results," Jun. 2020. [Online]. Available:

https://mepag.jpl.nasa.gov/meeting/2020-06/MASWG-preliminary-results-MEPAG-2 6June2020.pdf.

[2] J. Foust, "Astrobotic wins NASA contract to deliver VIPER lunar rover," SpaceNews, Jun. 11, 2020.

[3] P. B. Niles et al., "Multiple Smaller Missions as a Direct Pathway to Mars Sample Return," vol. 1679, p. 4234, Jun. 2012. 